\title{
Comparison of Fuzzy Multiplication Operation on Triangular Fuzzy Number
}

\author{
Md. Yasin Ali ${ }^{1}$, Abeda Sultana ${ }^{2}$ and A F M Khodadad Khan ${ }^{3}$ \\ ${ }^{I}$ School of Science and Engineering, University of Information Technology \& Sciences, Baridhara, Dhaka, \\ Bangladesh \\ ${ }^{2}$ Department of Mathematics, Jahangirnagar University, Savar, Bangladesh \\ ${ }^{3}$ School of Engineering and Computer Science, Independent University Bashundhara R/A, Dhaka, Bangladesh
}

\begin{abstract}
The arithmetic operations on fuzzy number are basic content in fuzzy mathematics. But still the operations of fuzzy arithmetic operations are not established. There are some arithmetic operations for computing fuzzy number. Certain are analytical methods and further are approximation methods. In this paper we, compare the multiplication operation on triangular fuzzy number between $\alpha-c u t$ method and standard approximation method and give example and graphical representation supporting each example.
\end{abstract}

Keywords: Fuzzy number, Triangular Fuzzy number, $\alpha$-cut method, Standard approximation method.

\section{Introduction}

Fuzzy sets have been introduced by Lotfi.A.Zadeh(1965)[1].Since its inception 50 years ago, the theory of fuzzy sets has advanced in a variety of ways and in many disciplines. Applications of this theory can be found, for example, in artificial intelligence, computer science, control engineering, decision theory, expert systems, logic, management science, operations research, pattern recognition, and robotics. Theoretical advances have been made in many directions. In application fuzzy set theory fuzzy number plays an important role. Arithmetic operations on fuzzy numbers have also been developed, and are based mainly on the extension principle [3] or interval arithmetic [4]. When operating with fuzzy numbers, the result of our calculations strongly depend on the shape of the membership functions of these numbers. Less consistent membership functions lead to more complicated calculations. Moreover, fuzzy numbers with simpler shape of membership functions often have more intuitive and more natural interpretation. Considering the interval arithmetic -based arithmetic operations on triangular fuzzy numbers, the product of two such fuzzy numbers is not of the same kind: the shape of these fuzzy numbers is not preserved. In many situations this problem is solved by approximation multiplication by a triangular or trapezoidal fuzzy number. In this paper we mentioned interval arithmetic operation as $\alpha$-cut method and approximation multiplication as standard approximation and compare them over triangular fuzzy number.

The paper is organized as follows: in section 2 we discuss some basic definitions, in section 3 , we discuss $\alpha$-cut method and standard approximation. In section 4 , we discuss error analysis between $\alpha$-cut method and standard approximation and in section 5 numerical example and graphical result are shown for different cases.

\section{Preliminaries}

2.1 Fuzzy set: A fuzzy set $\widetilde{A}$ is defined by $\tilde{A}=\left\{\left(x, \mu_{A}(x)\right): x \in A, \mu_{A}(x) \in[0,1]\right\}$. In the pair $\left(x, \mu_{A}(x)\right)$, the first element $x$ belong to the classical set $A$, the second element $\mu_{A}(x)$, belong to the interval [0,1], called Membership function.

2.2 Support of Fuzzy Set: The support of fuzzy set $\tilde{A}$ is the set of all points $x$ in $X$ such that $\mu_{\tilde{A}}>0$. That is Support $(\tilde{A})=\left\{x \mid \mu_{\tilde{A}}(x)>0\right\}$.

$2.3 \alpha$ - cut: The $\alpha$ - cut of $\alpha$ - level set of fuzzy set $\tilde{A}$ is a set consisting of those elements of the universe $X$ whose membership values exceed the threshold level $\alpha$. That is $\tilde{A}_{\alpha}=\left\{x \mid \mu_{\tilde{A}}(x) \geq \alpha\right\}$. 
2.4 Fuzzy Number: A fuzzy set $\widetilde{A}$ on $\mathrm{R}$ must possess at least the following three properties to qualify as a fuzzy number,

(i) $\tilde{A}$ must be a normal fuzzy set;

(ii) $\tilde{A}_{\alpha}$ must be closed interval for every $\alpha \in[0,1]$

(iii) the support of $\tilde{A}, \tilde{A}_{0+}$, must be bounded.

\subsection{Triangular Fuzzy Number:}

It is a fuzzy number represented with three points as follows: $\tilde{A}=\left(a_{1}, a_{2}, a_{3}\right)$.This representation is interpreted as membership functions and holds the following conditions

(i) $a_{1}$ and $a_{2}$ is increasing function

(ii) $a_{2}$ and to $a_{3}$ is decreasing function

(iii) $a_{1} \leq a_{2} \leq a_{3}$.

$\mu_{\tilde{A}(x)}=\left\{\begin{array}{cc}0 & , x<a_{1} \\ \frac{x-a_{1}}{a_{2}-a_{1}} & , \quad a_{1} \leq x \leq a_{2} \\ \frac{a_{3}-x}{a_{3}-a_{2}} & , \quad a_{2} \leq x \leq a_{3} \\ 0 & , \quad x>a_{3}\end{array}\right.$

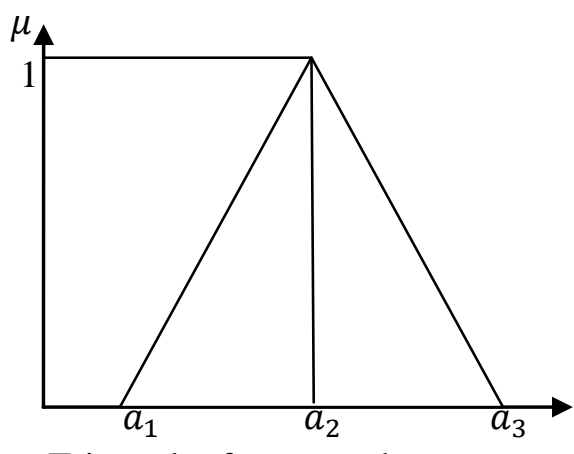

Triangular fuzzy number

2.6 $\alpha$ - cut of a triangular fuzzy number: We get a crisp interval by $\alpha$-cut operation; interval $A_{\alpha}$ shall be obtained as follows $\forall \alpha \in[0,1]$.

Thus $A_{\alpha}=\left[a_{1}^{\alpha}, a_{1}^{\alpha}\right]=\left[\left(a_{2}-a_{1}\right) \alpha+a_{1},-\left(a_{3}-a_{2}\right) \alpha+a_{3}\right]$

2.7 Positive triangular fuzzy number: A positive triangular fuzzy number $\tilde{A}$ is denoted as $\tilde{A}=$ $\left(a_{1}, a_{2}, a_{3}\right)$, where all $a_{i}$ 's $>0$ for all $i=1,2,3$.

2.8 Negative triangular fuzzy number: A negative triangular fuzzy number $\tilde{A}$ is denoted as $\tilde{A}=$ $\left(a_{1}, a_{2}, a_{3}\right)$, where all $a_{i}$ 's $<0$ for all $i=1,2,3$.

2.9 Partial Negative triangular fuzzy number: A Partial Negative triangular fuzzy number $\tilde{A}$ is denoted as $\tilde{A}=\left(a_{1}, a_{2}, a_{3}\right)$, where at least one $a_{i}$ is negative for all $i=1,2,3$.

\section{III. $\quad \alpha-$ cutMethodand Standard Approximation}

The Triangular fuzzy numbers (TFN) shape is not closed under multiplication and division. The result of these operators is a polynomial membership function and the triangular shape only approximates the actual result. The operators of fuzzy addition and subtraction are closed and the definitions provided in Table 1are exact. Fuzzy multiplication and division are not closed; the definitions in Table 1 are only approximations to the actual results.

Table 1: Standard approximate arithmetic operations on TFNs and their definition for two fuzzy numbers $\tilde{A}$ and $\tilde{B}$ :

\begin{tabular}{|c|c|}
\hline Arithmetic operations & Definition \\
\hline$\tilde{A} \oplus \tilde{B}=$ & $<a_{1}+a_{2}, b_{1}+b_{2}, c_{1}+c_{2}>$ \\
$\tilde{A} \ominus \tilde{B}=$ & $<a_{1}-c_{2}, b_{1}-b_{2}, c_{1}-a_{2}>$ \\
$\tilde{A} \otimes \tilde{B}=$ & $<\min \left(a_{1} a_{2}, a_{1} c_{2}, c_{1} a_{2}, c_{1} c_{1}\right), b_{1} b_{2}, \max \left(a_{1} a_{2}, a_{1} c_{2}, c_{1} a_{2}, c_{1} c_{1}\right)>$ \\
$\tilde{A}=\tilde{A} \cdot \frac{1}{\tilde{B}}$, where $\frac{1}{\tilde{B}}=$ & $<\min \left(\frac{1}{a_{2}}, \frac{1}{b_{2}}, \frac{1}{c_{2}}\right), \operatorname{medin}\left(\frac{1}{a_{2}}, \frac{1}{b_{2}}, \frac{1}{c_{2}}\right), \max \left(\frac{1}{a_{2}}, \frac{1}{b_{2}}, \frac{1}{c_{2}}\right)>$ \\
\hline
\end{tabular}

Using the definition for multiplication in table 1, the product of two TFNs,

$\tilde{A} \rightarrow<a_{1}, b_{1}, c_{1}>$ and $\tilde{B} \rightarrow<a_{2}, b_{2}, c_{2}>$ is 
$\tilde{C}=\tilde{A} \otimes \tilde{B} \rightarrow<\min \left(a_{1} a_{2}, a_{1} c_{2}, c_{1} a_{2}, c_{1} c_{1}\right), b_{1} b_{2}, \max \left(a_{1} a_{2}, a_{1} c_{2}, c_{1} a_{2}, c_{1} c_{1}\right)>$

Expression (3.1) will be called the standard approximation. The actual result is found by two ways (i) $\alpha$-cut method and (ii) Extension principle method.

\section{1. $\alpha$ - cut Method}

The actual result is found by rewriting the membership function to define a set of closed intervals as in expression (2.1) .Then the expressions defining the closed intervals are operated on using interval arithmetic [5].

Case 3.1.1: For two positive fuzzy numbers

The product can be calculated,

$$
\begin{aligned}
\tilde{A} & =<a_{1}, b_{1}, c_{1}>\rightarrow\left[\left(b_{1}-a_{1}\right) \alpha+a_{1},-\left(c_{1}-b_{1}\right) \alpha+c_{2}\right] \\
& =\left[s_{1}, \bar{s}_{1}\right] \quad[\text { Say }] \\
\tilde{B} & =<a_{2}, b_{2}, c_{2}>\rightarrow\left[\left(b_{2}-a_{2}\right) \alpha+a_{2},-\left(c_{2}-b_{2}\right) \alpha+c_{2}\right] \\
& =\left[s_{2}, \bar{s}_{2}\right] \quad[\text { Say }]
\end{aligned}
$$

$$
\begin{aligned}
\tilde{C} & =\tilde{A} \otimes \tilde{B} \\
& =\left[\min \left(s_{1} s_{2}, s_{1} \bar{s}_{2}, \bar{s}_{1} s_{2}, \bar{s}_{1} \bar{s}_{2}\right), \max \left(s_{1} s_{2}, s_{1} \bar{s}_{2}, \bar{s}_{1} s_{2}, \bar{s}_{1} \bar{s}_{2}\right)\right] \text { for } \alpha \in(0,1]
\end{aligned}
$$

Case 3.1.2: When any $\tilde{A}$ and $\tilde{B}$ is partial negative and other is positive fuzzy number, the product of $\tilde{A} \otimes \tilde{B}$ can't obtain according to the expression (3.2). The interval of $\alpha \in(0,1]$ will be divided into two parts, according to the intersection point of the two minimum expression in the $\alpha$-cut of $\tilde{C}$. Let us this intersection point is $\alpha_{s}$. Then

$$
\begin{aligned}
\tilde{C} & =\tilde{A} \otimes \tilde{B} \\
& =\left[\begin{array}{l}
\min \left(s_{1} s_{2}, s_{1} \bar{s}_{2}, \bar{s}_{1} s_{2}, \bar{s}_{1} \bar{s}_{2}\right), \max \left(s_{1} s_{2}, s_{1} \bar{s}_{2}, \bar{s}_{1} s_{2}, \bar{s}_{1} \bar{s}_{2}\right) \text { for } \alpha \in\left(0, \alpha_{s}\right] \\
\min \left(s_{1} s_{2}, s_{1} \bar{s}_{2}, \bar{s}_{1} s_{2}, \bar{s}_{1} \bar{s}_{2}\right), \max \left(s_{1} s_{2}, s_{1} \bar{s}_{2}, \bar{s}_{1} s_{2}, \bar{s}_{1} \bar{s}_{2}\right) \text { for } \alpha \in\left(\alpha_{s}, 1\right]
\end{array}\right]
\end{aligned}
$$

Case 3.1.3: When $\tilde{A}$ is negative and $\tilde{B}$ is positive fuzzy number. Then the multiplication of $\tilde{A}$ and $\tilde{B}$ can be found as expression (3.2).

Case 3.1.4: When $\tilde{A}$ and $\tilde{B}$ both are negative fuzzy number. Then the multiplication of $\tilde{A}$ and $\tilde{B}$ can also be found as expression (3.2).The expression (3.2) and (3.3) are known as analytical method for fuzzy arithmetic operation. The standard approximation and actual results are shown in figure $(1,2,3$, and 4$)$. In actual product the lines connecting the ends points are parabolic and in standard approximation lines connecting the ends points are triangular form.

\section{Error analysis for $\alpha$ - cut Method}

The error is the difference at a given $\alpha$ - level, between the approximated membership function and exact results in the expression (3.2) and (3.3). Each TFN can be separated in to left and right segments in accordance with the LR parametered representation. The actual product of (3.2) and (3.3) will have the value $x$ at a given $\alpha$ defined as $T_{L}$ for the left segment, and $T_{R}$ for the right segment. The standard approximation (3.1) will have a value $x$, at a given $\alpha$ defined as $P_{L}$ and $P_{R}$ for the left and right segments respectively.

This follows us to separately analyses the left and right portions of the membership curve .The left and right segment error are then.

$E_{L}=P_{L}-T_{L}$ and $E_{R}=P_{R}-T_{R}$.

Graphically this is the horizontal distance between the two curves as shown in Figure (1, 2, 3, and 4) and numerical error is shown in table (2, 3, 4 and 5).

\section{Numerical Experiment}

Example 5.1: We consider two positive TFNs as

$$
\tilde{A}(x)=\left\{\begin{array}{cc}
x-2, & 2 \leq x \leq 3 \\
\frac{-x}{2}+\frac{5}{2}, & 3 \leq x \leq 5
\end{array}\right.
$$


Here, $A_{\alpha}=[2+\alpha, 5-2 \alpha], B_{\alpha}=[2 \alpha+3,-\alpha+6]$

$$
\tilde{B}(y)=\left\{\begin{array}{cc}
\frac{y}{2}-\frac{3}{2}, & 3 \leq y \leq 5 \\
-y+6, & 5 \leq y \leq 6
\end{array}\right.
$$

$$
(\tilde{A} \otimes \tilde{B})_{\alpha}=\left[2 \alpha^{2}+7 \alpha+6,2 \alpha^{2}-17 \alpha+30\right]
$$

The membership functions of $\tilde{A} \otimes \tilde{B}$ in $\alpha-$ cut method as follows:

$$
\tilde{A} \otimes \tilde{B}(z)= \begin{cases}\frac{-7}{4}+\frac{\sqrt{1+8 z}}{4}, & 6 \leq z \leq 15 \\ \frac{17}{4}-\frac{\sqrt{49+8 z}}{4}, & 15 \leq z \leq 30\end{cases}
$$

The membership functions in standard approximation are:

$$
\tilde{A} \otimes \tilde{B}(z)=\left\{\begin{array}{cc}
\frac{z}{9}-\frac{2}{3}, & 6 \leq z \leq 15 \\
\frac{-z}{15}+2, & 15 \leq z \leq 30
\end{array}\right.
$$

Table 2: Comparison of $\alpha-$ cut Method and Standard Approximation when both are positive TFNs:

\begin{tabular}{|l|l|l|l|l|l|l|}
\hline \multirow{2}{*}{ Value of $\alpha$} & \multicolumn{2}{l}{ Actual Product } & \multicolumn{2}{l|}{ Standard Approximation } & \multicolumn{2}{l|}{ Error } \\
\cline { 2 - 7 } & Left & Right & Left & Right & Left & Right \\
\hline 1 & 15 & 15 & 15 & 15 & 0 & 0 \\
\hline 0.9 & 13.92 & 16.32 & 14.1 & 16.5 & 0.18 & 0.18 \\
\hline 0.8 & 12.88 & 17.68 & 13.2 & 18 & 0.32 & 0.32 \\
\hline 0.7 & 11.88 & 19.08 & 12.3 & 19.5 & 0.42 & 0.42 \\
\hline 0.6 & 10.92 & 20.52 & 11.4 & 21 & 0.48 & 0.48 \\
\hline 0.5 & 10 & 22 & 15.5 & 22.5 & 0.5 & 0.5 \\
\hline 0.4 & 9.12 & 23.52 & 9.6 & 24 & 0.48 & 0.48 \\
\hline 0.3 & 8.28 & 25.08 & 8.7 & 25.5 & 0.42 & 0.42 \\
\hline 0.2 & 7.48 & 26.68 & 7.8 & 27 & 0.32 & 0.32 \\
\hline 0.1 & 6.72 & 28.32 & 6.9 & 28.58 & 0.18 & 0.18 \\
\hline 0 & 6 & 30 & 6 & 30 & 0 & 0 \\
\hline
\end{tabular}

Example 5.2: When one is partial negative and another is positive TFNs as:

$$
\begin{gathered}
\tilde{A}(x)= \begin{cases}\frac{x}{2}+\frac{1}{2}, & -1 \leq x \leq 1 \\
\frac{3}{2}-\frac{x}{2}, & 1 \leq x \leq 3\end{cases} \\
\tilde{B}(y)= \begin{cases}\frac{y}{2}-\frac{1}{2}, & 1 \leq y \leq 3 \\
\frac{5}{2}-\frac{y}{2}, & 3 \leq y \leq 5\end{cases}
\end{gathered}
$$

Here, $A_{\alpha}=[2 \alpha-1,3-2 \alpha], B_{\alpha}=[2 \alpha+1,-2 \alpha+5]$

$(\tilde{A} \otimes \tilde{B})_{\alpha}=\left\{\begin{array}{c}{\left[-4 \alpha^{2}+12 \alpha-5,4 \alpha^{2}-16 \alpha+15\right] \text { for } \alpha \in(0, .5]} \\ {\left[4 \alpha^{2}-1,4 \alpha^{2}-16 \alpha+15\right] \text { for } \alpha \in(0.5,1]}\end{array}\right.$

The membership functions of $\tilde{A} \otimes \tilde{B}$ in $\alpha-$ cut method as follows:

The membership functions in standard approximation are:

$$
\tilde{A} \otimes \tilde{B}(z)= \begin{cases}\frac{3-(4-z)^{\frac{1}{2}}}{2} & ,-5 \leq z<0 \\ \frac{(1+z)^{\frac{1}{2}}}{2} & , 0 \leq z<3 \\ \frac{4-(1+z)^{\frac{1}{2}}}{2} & , 3 \leq z \leq 15\end{cases}
$$




$$
\tilde{A} \otimes \tilde{B}(z)=\left\{\begin{array}{lc}
\frac{z}{8}+\frac{5}{8}, & -5 \leq z \leq 3 \\
\frac{-z}{15}+\frac{5}{4}, & 3 \leq z \leq 15
\end{array}\right.
$$

Table 3: Comparison when $\tilde{A}$ is partial negative and $\tilde{B}$ is positive triangular fuzzy numbers:

\begin{tabular}{|l|l|l|l|l|l|l|}
\hline \multirow{2}{*}{ Value of $\alpha$} & \multicolumn{2}{|c|}{ Actual Product } & \multicolumn{2}{c|}{ Standard Approximation } & \multicolumn{2}{c|}{ Error } \\
\cline { 2 - 8 } & Left & Right & Left & Right & Left & Right \\
\hline 1 & 3 & 3 & 3 & 3 & 0 & 0 \\
\hline 0.9 & 2.24 & 3.84 & 2.2 & 4.2 & -0.04 & 0.36 \\
\hline 0.8 & 1.56 & 4.76 & 1.4 & 5.4 & -0.16 & 0.64 \\
\hline 0.7 & 0.96 & 5.76 & 0.6 & 6.6 & -0.36 & 0.84 \\
\hline 0.6 & 0.44 & 6.84 & -0.2 & 7.8 & -0.46 & 0.96 \\
\hline 0.5 & 0 & 8 & -1 & 9 & -1 & 1 \\
\hline 0.4 & -0.84 & 9.24 & -1.8 & 10.2 & -0.96 & 0.96 \\
\hline 0.3 & -1.76 & 10.56 & -2.6 & 11.4 & -0.84 & 0.84 \\
\hline 0.2 & -2.76 & 11.96 & -3.4 & 12.6 & -0.64 & 0.64 \\
\hline 0.1 & -3.84 & 13.44 & -4.2 & 13.8 & -0.36 & 0.36 \\
\hline 0 & -5 & 15 & -5 & 15 & 0 & 0 \\
\hline
\end{tabular}

Example 5.3: We consider $\tilde{A}(x)$ negative and $\tilde{B}(x)$ positive TFNs as,

$$
\begin{gathered}
\tilde{A}(x)=\left\{\begin{array}{lc}
\frac{x+5}{2}, & -5 \leq x \leq-3 \\
-\frac{x+1}{2}, & -3 \leq x \leq-1
\end{array}\right. \\
\tilde{B}(y)=\left\{\begin{array}{cc}
y-1, & 1 \leq y \leq 2 \\
-y+3, & 2 \leq y \leq 3
\end{array}\right.
\end{gathered}
$$

The membership functions of $\tilde{A} \otimes \tilde{B}$ in $\alpha-$ cut method as follows:

The membership functions in standard approximation are:

$$
\tilde{A} \otimes \tilde{B}(z)= \begin{cases}\frac{11}{4}+\frac{\sqrt{41+8 z}}{4}, & -15 \leq z \leq-6 \\ \frac{-3}{4}-\frac{\sqrt{1-8 z}}{4}, & -6 \leq z \leq-1\end{cases}
$$

\begin{tabular}{|c|c|c|c|c|c|c|}
\hline \multirow[t]{2}{*}{ Value of $\alpha$} & \multicolumn{2}{|c|}{ Actual Product } & \multicolumn{2}{|r|}{ Approximation } & \multicolumn{2}{|c|}{ Error } \\
\hline & Left & Right & Left & Right & Left & Right \\
\hline 1 & -6 & -6 & -6 & -6 & 0 & 0 \\
\hline 0.9 & -6.72 & -5.32 & -6.9 & -5.5 & -0.18 & -0.18 \\
\hline 0.8 & -7.48 & -4.68 & -7.8 & -5 & -0.32 & -0.32 \\
\hline 0.7 & -8.28 & -4.08 & -8.7 & -4.5 & -0.42 & -0.42 \\
\hline 0.6 & -9.12 & -3.52 & -9.6 & -4 & -0.48 & -0.48 \\
\hline 0.5 & -10 & -3 & -10.5 & -3.5 & -0.5 & -0.5 \\
\hline 0.4 & -10.92 & -2.52 & -11.4 & -3 & -0.48 & -0.48 \\
\hline 0.3 & -11.88 & -2.08 & -12.3 & -2.5 & -0.42 & -0.42 \\
\hline 0.2 & -12.88 & -1.68 & -13.2 & -2 & -0.32 & -0.32 \\
\hline 0.1 & -13.92 & -1.32 & -14.1 & -1.5 & -0.18 & -0.18 \\
\hline 0 & -15 & -1 & -15 & -1 & 0 & 0 \\
\hline
\end{tabular}

$$
\tilde{A} \otimes \tilde{B}(z)=\left\{\begin{array}{cc}
\frac{z}{9}+\frac{15}{9}, & -15 \leq z \leq-6 \\
\frac{-z}{5}-\frac{1}{5}, & 6 \leq z \leq-1
\end{array}\right.
$$

Table 4: When $\tilde{A}(x)$ negative and $\tilde{B}(x)$ positive TFNs:

Example 5.4: We consider two negative TFNs as:

$$
\tilde{A}(x)= \begin{cases}\frac{x+3}{2}, & -6 \leq x \leq-4 \\ -\frac{x}{2}-1, & -4 \leq x \leq-2\end{cases}
$$




$$
\tilde{B}(y)=\left\{\begin{array}{l}
\frac{y}{2}+\frac{5}{2}, \quad-5 \leq y \leq-3 \\
\frac{-y}{2}-\frac{1}{2},-3 \leq y \leq-1
\end{array}\right.
$$

The membership functions of $\tilde{A} \otimes \tilde{B}$ in $\alpha-$ cut method as follows:

$$
\tilde{A} \otimes \tilde{B}(z)= \begin{cases}\frac{-3}{4}+\frac{\sqrt{1+4 z}}{4}, & 2 \leq z \leq 12 \\ \frac{11}{4}+\frac{\sqrt{1+8 z}}{16}, & 12 \leq z \leq 30\end{cases}
$$

The membership functions in standard approximation are:

$$
\tilde{A} \otimes \tilde{B}(z)= \begin{cases}\frac{z-2}{10}, & 2 \leq z \leq 12 \\ \frac{30-z}{18}, & 12 \leq z \leq 30\end{cases}
$$

Table 5: When $\tilde{A}$ and $\tilde{B}$ both are negative TFNs as:

\begin{tabular}{|l|l|l|l|l|l|l|}
\hline \multirow{2}{*}{ Value of $\alpha$} & \multicolumn{2}{|c|}{ Actual Product } & \multicolumn{2}{c|}{ Standard } & Approximation & \multicolumn{2}{c|}{ Error } \\
\cline { 2 - 8 } & Left & Right & Left & Right & Left & Right \\
\hline 1 & 12 & 12 & 12 & 12 & 0 & 0 \\
\hline 0.9 & 10.64 & 13.44 & 11 & 13.8 & 0.36 & 0.36 \\
\hline 0.8 & 9.36 & 14.96 & 10 & 15.6 & 0.64 & 0.64 \\
\hline 0.7 & 8.16 & 16.56 & 9 & 17.4 & 0.84 & 0.84 \\
\hline 0.6 & 7.04 & 18.24 & 8 & 19.2 & 0.96 & 0.96 \\
\hline 0.5 & 6 & 20 & 7 & 21 & 1 & 1 \\
\hline 0.4 & 5.04 & 21.84 & 6 & 22.8 & 0.96 & 0.96 \\
\hline 0.3 & 4.16 & 23.76 & 5 & 24.6 & 0.84 & 0.84 \\
\hline 0.2 & 3.36 & 25.76 & 4 & 26.4 & 0.64 & 0.64 \\
\hline 0.1 & 2.64 & 27.84 & 3 & 28.2 & 0.36 & 0.36 \\
\hline 0 & 2 & 30 & 2 & 30 & 0 & 0 \\
\hline
\end{tabular}

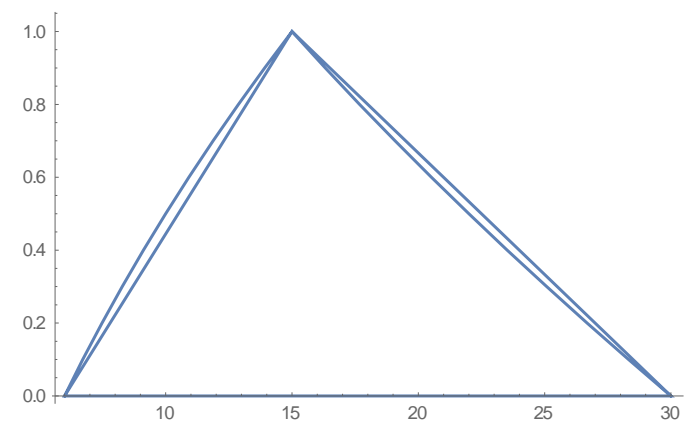

Fig.1 Comparison of $\alpha-$ cut Method and Standard Approximation when both are positive TFNs

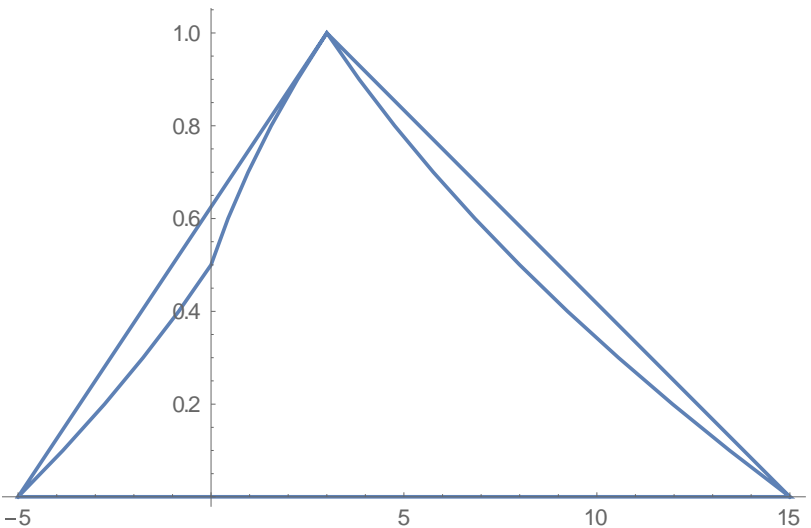

Fig.2 Comparison when $\tilde{A}$ is partial negative and $\tilde{B}$ is positive TFNs 


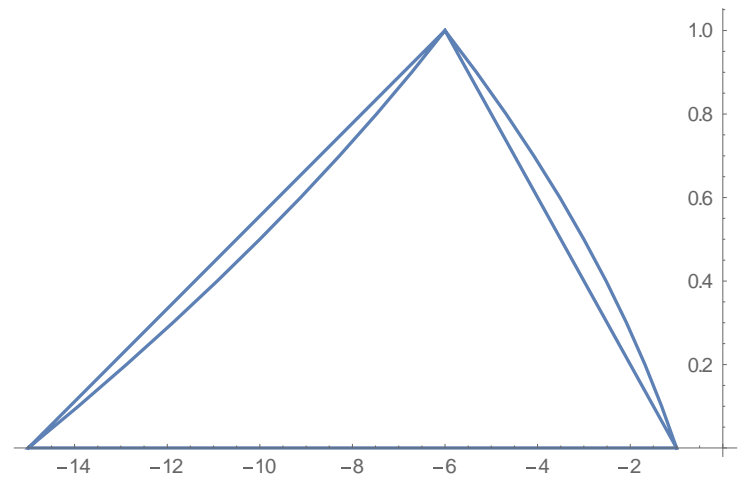

Fig.3 Comparison when $\tilde{A}$ is negative and $\tilde{B}$ is positive TFNs

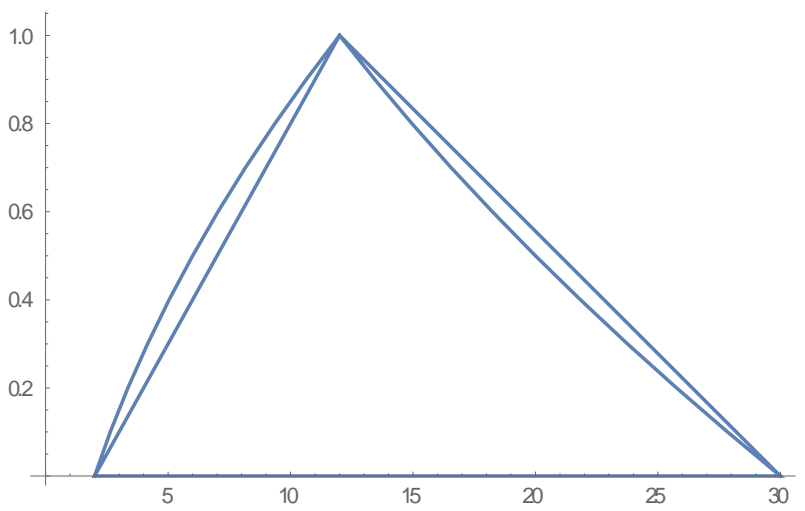

Fig.4 Comparison when both are negative TFNs

\section{Conclusion}

Here we have tried to compare the fuzzy arithmetic operation on triangular fuzzy number between analytical method and approximation method. Since fuzzy arithmetic operations play an important role in different field of sciences and engineering, so any one can get idea about the result between analytical method and approximation. We also represent these results graphically.

\section{References}

[1]. $\quad$ L. A. Zadeh, "Fuzzy set", Information and Control, vol.8, no.3, 338-353, 1965.

[2]. Dubois.D and Prade.H,Operations on fuzzy numbers,International Journal of Systems Science, vol.9, no.6, 613-626, 1978

[3]. Rogers, F., Jun, Y., Fuzzy Nonlinear Optimization for the Linear Fuzzy Real Number System, International Mathematical Forum, 4(12), 2009, $587-596$.

[4]. R. E. Moore, Methods and Applications of Interval Analysis(SIAM, Philadelphia, 1979)

[5]. Abeda Sultana, UtpalenduDev and NirmalKantimitra, Algebra of intervals, J. Math. and Math. Sci., Vol. 23, 2008, 49-62.

[6]. KlirG.J.,Yuan B., Fuzzy sets and fuzzy logic Theory and Applications, (Prentice-Hall of India Private Limited, New Delhi, (1997), P.105- 107)

[7]. A. NagoorGani, S. N. Mohamed Assarudeen,Solving Fuzzy Linear ProgrammingProblem,Applied Mathematical Sciences, Vol. 6,2012 , no. $11,525-532$.

[8]. A. Kaufman M. M. Gupta, Introduction to fuzzy arithmetic, theory and applications(Van Nostrand Reinhold Company, New York, 1985).

[9]. Guanrong Chen, Trung Tat Pham, Introduction to fuzzy sets, fuzzy logic, and fuzzy control systems, (Printed by LCC press, in the United States of America, 2001). 